\title{
EL DOCENTE COMO MEDIADOR: FUNDAMENTOS, CARACTERÍSTICASE IMPORTANCIA EN LOS ENTORNOS VIRTUALES DE APRENDIZAJE
}

\author{
THE TEACHER AS A MEDIATOR: \\ FOUNDATION, CHARACTERISTICS AND IMPORTANCE IN VIRTUAL LEARNING \\ ENVIRONMENTS
}

\author{
Marymili Segura Vera ${ }^{1}$ \\ Magister en Educación \\ Docente de Maestría \\ Universidad de la Empresa, Montevideo Uruguay \\ marymilisegura@gmail.com
}

\begin{abstract}
Resumen: La dinámica social de los últimos tiempos ha conllevado a cambios en el proceso educativo que a raíz de la pandemia de Covid-19 se han evidenciado muchos más, resaltando la importancia de los roles del docente en los entornos virtuales de aprendizaje, cobrando especial importancia su papel como mediador, en el que su apoyo y disponibilidad deben manifestarse ante cualquier duda o dificultad que pueda surgir en sus estudiantes. De aquí surge el interés de este estudio cuyo objetivo es analizar los aspectos que caracterizan al docente como mediador en los entornos virtuales de aprendizaje. Se desarrolló una investigación de tipo documental, con un nivel descriptivo. Entre las reflexiones finales cabe resaltar que más allá de los avances tecnológicos, la mediación docente es la que define el éxito de los procesos pedagógicos mediados por tecnologías. Tener definida la intencionalidad del curso que se está impartiendo, seleccionar contenidos y actividades con significado para los participantes y guiar a los estudiantes para que los conocimientos adquiridos trasciendan más allá del momento y el contexto educativo son factores fundamentales que se logran a través de la mediación y que deben estar presente como parte de la dinámica en los entornos virtuales de aprendizaje.
\end{abstract}

Palabras Clave: Educación Virtual, profesor Mediador, Mediación en entornos Virtuales de Aprendizaje, Práctica Docente y TIC, Rol Docente

Resumo: A dinâmica social dos últimos tempos tem levado a mudanças no processo educacional que, em decorrência da pandemia de Covid-19, muitas mais foram evidenciadas, destacando-se a importância do papel do professor em ambientes virtuais de aprendizagem, com especial importância sendo seu papel como mediador, no qual o seu apoio e disponibilidade deverão manifestar-se face a qualquer dúvida ou dificuldade que possa surgir nos seus alunos. Disto surge o interesse deste estudo cujo objetivo é analisar os aspectos que

\footnotetext{
${ }^{1}$ Magister en Educación de la Universidad de Carabobo. Docente de Nuevas Tecnologías y Educación en la
} Maestría en Educación de la Universidad de la Empresa. 
caracterizam o professor como mediador em ambientes virtuais de aprendizagem. Foi desenvolvida uma investigação do tipo documental, com nível descritivo. Entre as reflexões finais, cabe destacar que, para além dos avanços tecnológicos, a mediação docente é o que define o sucesso dos processos pedagógicos mediados pelas tecnologias. Definir a intencionalidade do curso a ser ministrado, selecionar conteúdos e atividades com sentido para os participantes e orientar os alunos para que os conhecimentos adquiridos transcendam o momento e o contexto educacional são fatores fundamentais que se alcançam por meio da mediação e que devem estar presentes como parte da dinâmica em ambientes virtuais de aprendizagem.

Palavras-chave: Educação Virtual, Professor Mediador, Mediação em Ambientes Virtuais de Aprendizagem, Prática de Ensino e TIC, Rol Docente

Abstract: The social dynamics of recent times have led to changes in the educational process that because of the Covid-19 pandemic, many more have been evidenced, highlighting the importance of the teacher's roles in virtual learning environments, with special importance being their role as a mediator, in which their support and availability should be manifested in the face of any doubt or difficulty that may arise in their students. From this arises the interest of this study whose objective is to analyze the aspects that characterize the teacher as a mediator in virtual learning environments. A documentary-type investigation was developed, with a descriptive level. Among the final reflections, it should be noted that beyond technological advances, teacher mediation is what defines the success of pedagogical processes mediated by technologies. Defining the intentionality of the course being taught, selecting content and activities with meaning for the participants and guiding the students so that the knowledge acquired transcends beyond the moment and the educational context are fundamental factors that are achieved through mediation and that they must be present as part of the dynamics in virtual learning environments.

Keywords: Virtual Education, Mediator teacher, Mediation in Virtual Learning environments, Teaching Practice and ICT, Teaching Role

\section{INTRODUCCIÓN}

La educación, por su esencia humanística, se mantiene en continuos cambios derivado de la necesidad de adaptarse a la dinámica social que caracteriza cada época. En los últimos tiempos esta dinámica ha girado alrededor de las tecnologías de información y comunicación (TIC), observándose una acentuación de la intervención de estas tecnologías en los procesos de enseñanza y aprendizaje en todos los niveles y contextos educativos. Siendo esta realidad más evidente en el nivel universitario, se observa cada vez con más frecuencia la oferta de programas de grado o de posgrado parcial o totalmente desarrollados en Entornos Virtuales de Aprendizaje (desde ahora EVA), los cuales dan paso a la inclusión y apertura educativa, pues son eliminadas dos grandes barreras: distancia y tiempo. 
Másallá de las particularidades de cada curso y de cada contexto, en los entornos virtuales de aprendizaje es muy importante la presencia activa del docente, quien a través de diversas estrategias debe manifestar su apoyo y disponibilidad ante cualquier duda o dificultad que pueda surgir en el proceso de aprendizaje. Aunque se podría suponer que esto es algo evidente, ya que es inherente al rol docente fortalecer el nexo profesor-estudiante o disminuir lo que Moore (1997) denomina distancia transaccional ${ }^{2}$, no siempre se logra con facilidad. El hecho de que un participante indique, por ejemplo, en el foro inicial quien es y cuáles son sus expectativas no basta para crear el nexo con el profesor y con los compañeros. Con frecuencia se hace necesario que el docente desarrolle estrategias que impulsen el dialogo y el intercambio, promover las intervenciones y la interacción entre compañeros, es lo que se conoce como moderar o mediar un espacio virtual.

Ante todos los cambios pedagógicos que han surgido a raíz de la pandemia del Covid-19, los docentes en general han tenido que adaptarse y reinventarse drásticamente para poder atender las nuevas demandas educativas. Esto ha significado grandes esfuerzos tanto para lograr el dominio de las herramientas tecnológicas como en la planificación de nuevas estrategas didácticas adaptadas a este contexto. Sin embargo y a pesar de que los entes encargados han dispuesto los mecanismos necesarios para la formación docente en el uso de las TIC, con el propósito llevar adelante la virtualización de la educación en este momento coyuntural, en la mayoría de los casos han dejado de lado otros elementos de suma importancia, como lo es la mediación docente. Situación que en este contexto de pandemia se ha evidenciado observándose con frecuencia grandes debilidades comunicativas y de mediación que aún existen en los entornos virtuales de aprendizaje.

Por todo lo señalado anteriormente surge el interés de analizar los aspectos que caracterizan al docente como mediador en los entornos virtuales de aprendizaje, para lo cual se realizó una investigación enmarcada en un diseño bibliográfico, de tipo documental, con un nivel descriptivo. En la cual se inicia con una aproximación a la realidad de los entornos virtuales de aprendizaje y la mediación docente, se describen los aspectos que denotan a la comunicación como la esencia de la mediación, detallando seguidamente los criterios de mediación universales y su aplicabilidad en los entornos virtuales de aprendizaje, Finalizando con la descripción de las actividades de mediación en estos entornos mediados por tecnologías.

\section{APROXIMÁNDONOS A LA REALIDAD ACTUAL SOBRE MEDIACIÓN EN ENTORNOS VIRTUALES}

Más allá de los grandes beneficios que enmarcan a esta modalidad de estudio, uno de los problemas más frecuentes en programas o cursos desarrollados en entornos virtuales es la pérdida de entusiasmo o motivación de los participantes la cual en muchos casos deriva en el abandono del curso. En este sentido, Taylor-Guy y Chase (2020) señalan que las tasas de

\footnotetext{
2 Es la separación de alumnos y profesores lo que afecta profundamente a la enseñanza y el aprendizaje. Cuando Moore (1997) habla de distancia transaccional se refiere espacios psicológicos y de comunicaciones, en los que potencialmente podrían surgir malentendidos entre las aportaciones del instructor y las del alumno
} 
finalización de los alumnos que estudian totalmente en línea en muchos países son considerablemente más bajas que las de los que estudian modalidad presencial, resaltando que, en Australia, la deserción es al menos un $20 \%$ más alta para los estudiantes en línea en comparación con los que asisten al campus y la titulación de los estudiantes en línea es 2.5 veces menor. En el caso de España, para el periodo 2018-2019, la tasa de abandono de estudio a nivel de grado en universidades no presenciales fue de $62.1 \%$, mientras que la tasa de abandono a nivel de máster fue del 30,8\%.

En el caso de América latina, la educación universitaria en línea y los entornos virtuales de aprendizaje han aumentado significativamente. Según datos de la UNESCO y la Fundación Carolina en el 2017 los estudiantes inscritos en esta modalidad llegaron a 4,3 millones, siendo los países con mayor penetración Brasil, Colombia y México (Fernández, 2020). Este contexto no escapa de la problemática de altos índices de deserción. Una investigación realizada por el Laboratorio de Economía de la Educación de la Universidad Javeriana de Colombia señala que las carreras de pregrado mediadas por tecnologías tienen una tasa de deserción dos veces más alta que las carreras presenciales, Ilegaron a esa conclusión al hacer un análisis de la evolución en las matrículas y programas académicos de la modalidad virtual en el país entre 2011 y 2018 (El tiempo, 2020)

En este orden de ideas, Frankola (2001) resalta entre las razones asociadas a la decisión de abandono de los cursos en línea los problemas tecnológicos, falta de apoyo, cursos mal diseñados e instructores sin experiencia o incompetentes. Por su parte, García (2019) manifiesta que cuando se produce algún tipo de quiebre en la relación dialógica entre los agentes y componentes que interactúan en el proceso de enseñanza-aprendizaje (estudiantes, profesores, institución, recursos), el sistema no fluye adecuadamente o muestra debilidades que podrían llegar a la ruptura final del estudiante con la institución.

Como se puede observar, son varios los factores que pueden desencadenar esta ruptura, siendo uno de éstos la comunicación y el apoyo que el docente proporciona a los participantes. Si bien es cierto que los EVA deben ser espacios centrados en el alumno y caracterizados por la implementación de metodologías activas, también es cierta la relevancia del docente en su rol de gestor del conocimiento y como "mediador orientado hacia el aprendizaje en lugar de la enseñanza, fomentando la participación colaboración, cooperación, la creatividad, la reflexión, el análisis y la crítica" (Silva, 2017, p. 7).

Al respecto, en un estudio realizado, por Soto et al (2015) se pudo observar que el $14 \%$ de los encuestados manifestó como principal razón por la que abandonaron o no finalizaron un curso virtual la poca comunicación con el tutor. Estos autores concluyeron que, siendo la comunicación con los tutores asíncrona, las demoras en las respuestas a los participantes hacen que éstos sientan que la presencia de la persona tutora es escasa y en ocasiones nula y por este motivo las personas deciden abandonar el curso virtual. 


\section{LA COMUNICACIÓN, ESENCIA DE LA MEDICACIÓN}

Entendiendo que la educación es un proceso social y en consecuencia la comunicación es el elemento primordial de éste, se hace necesario mantenerla activa y fluida en sus diversas formas, adaptándola al medio y a los interlocutores. Para que esta comunicación genere aprendizaje es necesario que, tanto estudiantes como docentes estén comprometidos mutuamente en el logro del objetivo formativo del curso en el que participan, creando así el sentimiento de pertenencia a una comunidad (Ruiz et al, 2016)

Sin embargo, la responsabilidad de que esto se logre exitosamente en el contexto educativo recae en gran medida sobre el docente, independientemente de la modalidad en la que se desarrolle el proceso educativo. Esto se logra en gran medida a través de la mediación. En tal sentido Escobar (2011), hablando de la mediación del aprendizaje, la define como "un proceso de interacción pedagógica; social, dialógico, lúdico, consciente, intencional, sistemático, destinado a generar experiencias de 'buen aprendizaje', que al tiempo que transmita conocimientos, posibilite el desarrollo de las potencialidades humanas en el ser, hacer, conocer y convivir" (p. 60). En el caso de la modalidad virtual, Valencia et al (2016) resaltan entre las habilidades transversales que debe poseer un docente, las habilidades vocacionales y de liderazgo, entendidas como "la disposición para la formación de personas, el manejo innovador y creativo de los recursos (...) así como la habilidad para generar impacto e influencia, escuchar, preguntar, explicar y comunicar de manera efectiva" (p. 13).

Por otra parte, los EVA proveen gran cantidad de herramientas para mantener la continua comunicación entre los distintos actores (docente-estudiante; estudiante- estudiante; docentedocente), así mismo esta comunicación puede hacerse de forma sincrónica o asincrónica, inclusive la versatilidad de estos espacios permite la interacción a través de medios externos a dichos entornos. Es función del profesor dar uso efectivo a esas herramientas teniendo en cuenta las posibles diferencias culturales presentes en los participantes y de esa manera, como señalan Folgado et al (2020), mantener activos los espacios colaborativos de sus alumnos, facilitar la comunicación, convirtiéndose así en un tutor que motiva la participación de todos los miembros y acompaña al grupo en el logro de los objetivos comunes.

\section{Criterios de mediación universales}

La mediación no es un proceso superficial o aleatorio, por lo cual no toda interacción del docente con sus estudiantes debe asumirse como mediación. En este sentido, Feuerstein ${ }^{3}$ (como

\footnotetext{
${ }^{3}$ Reuven Feuerstein (1921-2014) desarrolló varios estudios sobre estructuras y procesos cognitivos, entre sus aportes están La Teoría de la Modificabilidad Cognitiva Estructural y la Teoría de la Experiencia de Aprendizaje Mediatizado (EAM), Esta última plantea que para que se produzca el aprendizaje mediatizado Es necesario "un mediatizador responsable, afectivo, conocedor y competente para ser intermediario entre el mediatizado y la experiencia de aprendizaje mediatizado" (Orrú, 2003, p. 37)
} 
se citó en Lobos et al, 2017) plantea tres criterios de mediación universales, los cuales se mencionan a continuación contextualizándolos en los entornos virtuales de aprendizaje

El primero de los criterios es el de Mediación de la intencionalidad y reciprocidad, a través del cual el docente debe estar consciente del propósito final del proceso pedagógico que desarrolla y, como mediador, debe dar a conocer este propósito a sus estudiantes de manera que al tener conciencia del mismo estos se involucren logrando reciprocidad, mayor dedicación y atención a las tareas correspondientes. En los entornos virtuales, esta intencionalidad debe estar evidenciada a través de la presentación del curso, de sus objetivos, contenidos del mismo y las competencias adquiridas por quienes lo culminen. Y así mismo especificar el propósito de cada unidad. La reciprocidad se logra con la comunicación constante de forma grupal y cuando sea necesario de forma individual, teniendo en cuenta el contexto de cada participante

El segundo de los criterios, identificado como Mediación del significado, el cual se refiere los contenidos y actividades que el mediador debe presentar de forma que tengan sentido desde las realidades de los estudiantes, potenciando el razonamiento y el aprendizaje significativo. Las Tecnologías de información presentan innumerables herramientas que ayudan al docente a dar significado al proceso educativo, desde lo audiovisual hasta la gamificación, son muchas las alternativas para integrar al estudiante desde sus conocimientos previos al logro de los nuevos aprendizajes, queda de parte del docente adaptar, en la medida de los posible, estas herramientas a las diversidades ideológicas y culturales presentes en los participantes.

El tercero de los criterios de mediación universales es el de trascendencia. El mediador debe guiar al estudiante para que sus expectativas trasciendan más allá de la singularidad de la situación específica estudiada y se genere en él nuevas inquietudes que lo induzcan a extrapolar los conocimientos adquiridos y buscar nuevos significados. En la virtualidad esta mediación se logra en los espacios de dialogo y reflexión grupal, los cuales pueden ser sincrónicos o asincrónicos. La participación en estos espacios puede ser alta o no, sin embargo, en todo caso la presencia del docente se debe sentir, moderando las intervenciones, reforzando conceptos y motivando nuevas participaciones.

\section{ACTIVIDADES DE MEDIACIÓN EN ENTORNOS VIRTUALES DE APRENDIZAJE}

Los procesos educativos tienen aspectos esenciales que se mantienen más allá del contexto pedagógico en los que se generen, sin embargo, la forma en la que el docente gestiona los aprendizajes e interactúa con sus estudiantes debe adecuarse tanto a las características de cada grupo como al entorno en el que se desarrollan las actividades. En los entornos virtuales de aprendizaje hay cuatro grandes aspectos que el docente debe manejar en su rol de mediador:

Mantener informado al grupo. Un espacio virtual de aprendizaje debe ser fluido y mantener al día a sus participantes sobre diversos aspectos de interés común. Esto se puede lograr a través de diversas estrategias como emails, espacios alternos en redes sociales o a través 
de secciones propias del entorno virtual destinadas específicamente a dar información a los participantes. Estas últimas se pueden configurar de acuerdo a la intencionalidad del docente. Silva (2010), las define como "elementos permanentes" del EVA, concebidos como espacios transversales a los contenidos del espacio virtual, que proveen información y atención general a los participantes. Estas secciones son:

Novedades y Foro social. En la sección de novedades el docente provee información del curso como calendario, fechas importantes, cambios en programación, entre otros. Aunque esta información les llega a todos los participantes a su correo, es unilateral, solo el docente publica en este espacio, los estudiantes no pueden responder o colocar información acá. Mientras que el foro social es un espacio informal, dedicado al intercambio social, en el que los participantes presentan los temas y entre ellos mismos animan la discusión. Silva (2010) sugiere que la intervención del tutor en este foro sea solo al inicio, para dar la bienvenida. Es un espacio para romper el hielo y crear nexos entre los participantes

Dudas técnicas y uso de la plataforma. Estas son alternativas destinadas a apoyar a los participantes sobre el uso de la plataforma, así como a resolver inconvenientes técnicos que puedan surgir a lo largo del curso, la interacción se desarrolla entre todos los miembros, es decir, una duda puede ser aclarada por cualquier participante del curso ya sea tutor, personal técnico o estudiante. Por otra parte, el grupo debe tener posibilidad de obtener Ayuda para el uso de la plataforma, este espacio también es conocido como "preguntas frecuentes" ya que se responden las preguntas que comúnmente realizan los participantes. Generalmente se presenta como una serie de preguntas y al entrar a cada una de ellas, se presenta una respuesta clara y detallada, muchas veces complementada con video tutoriales.

Programa del curso. Donde se describe detalladamente el curso, su estructura, contenidos, objetivos, metodología, evaluación, así como cualquier otro aspecto relevante del mismo. Este programa debe estar accesible a los participantes desde el inicio del curso y en todo momento.

A través de estos elementos se garantiza a los estudiantes el acceso a la información, apoyo técnico, así como el contacto inicial entre los integrantes. Aunque el docente en los espacios mencionados solo informa, estos vienen a ser fundamentales para que a lo largo del curso el proceso de mediación sea realmente exitoso

Fomentar el diálogo y la reflexión. La realización sistemática de las actividades o tareas establecidas en un curso no siempre conllevan a que el aprendizaje sea significativo y trascienda más allá del espacio educativo, muchas veces es necesario afianzar el aprendizaje a través de las experiencias de vida de los participantes. Estos aportes se pueden lograr a través de encuentros sincrónicos en los que se de paso a la discusión sobre temas relevantes relacionados con las actividades realzadas, el docente a través de su mediación guiará el dialogo y procurará mantener la dinámica y la participación activa de los presentes. 
Esto también se logra de forma asincrónica, a través de los foros de discusión. De Armas et al (2017) definen esta herramienta como "un elemento estratégico para lograr una participación reflexiva, colaborativa, de intercambio entre todos sus integrantes, estas características hacen de los foros una de las actividades más utilizada por los docentes". Así mismo Ruiz et al (2016) resaltan que el foro motiva la interacción entre iguales logrando que el estudiante se perciba como protagonista de su proceso formativo, afianzándolo como parte del grupo. Por otra parte, permite al docente modificar su perspectiva transformando su rol de transmisor a mediador, guiando al estudiante en un proceso activo de aprendizaje en el que puede construir conocimiento con significado y sentido desde sus saberes previos (Veitya, 2016).

Este tipo de mediación asincrónica se ejerce en dos momentos:

El primer momento es antes de iniciar la discusión, ya que el docente debe configurar el foro de acuerdo a lo que espera lograr en sus estudiantes. Esta configuración va desde lo estructural (el tipo de foro y las normas de participación) hasta la acertada descripción del tema a desarrollar. Esto implica presentar el tema a discutir e indicar qué se espera de cada participante de una forma clara para todos los integrantes independientemente de sus individualidades.

El segundo momento es ya en la discusión, cuando los participantes hacen sus aportes. En esta fase es importante la intervención del docente reforzando, haciendo nuevos aportes, o redireccionando la discusión si ésta llega a salirse del tema planteado. Por muy dinámico e interactivo que sea un grupo, la ausencia de las intervenciones del docente se perciben y pueden llegar a desmotivar a los participantes. En este sentido Sánchez (2010) señala entre las desventajas de los foros virtuales la sensación de aislamiento que puede tener el estudiante al no recibir pronta respuesta a su intervención y la posibilidad de que el tema se desvíe, por lo que es fundamental la presencia del docente como moderador, el docente debe dedicar una cantidad de tiempo considerable en la conducción de estas actividades.

Los espacios de dialogo y reflexión grupal son tan esenciales para integrar a los participantes como para generar significados de los aprendizajes adquiridos, más aún en el contexto educativos mediados por tecnologías, en los que los integrantes de cada grupo con frecuencia tienen diferentes historias, ideologías, realidades que generan una fascinante diversidad de perspectivas. Por esta razón estos espacios deben ser planificados de una forma holística e integradora.

Motivar, dinamizar la participación. En el contexto educativo motivar implica estimular la voluntad de aprender. En lo que se refiere al docente, la motivación tiene como propósitos direccionar la atención del alumno y despertar su interés; inducir el deseo por aprender que lo llevará a esforzarse y se mantener la constancia; dirigir al estudiante para que su esfuerzo y constancia lo conduzcan al logro de fines apropiados. Cuando el docente da significado a las tareas asignadas y las enmarca en un fin determinado, logra inducir motivos en sus alumnos, de manera tal que éstos realizarán la actividad escolar con gusto y dedicación, comprendiendo así su utilidad personal y social. Esto es lo que se conoce como motivación por el aprendizaje (DíazBarriga y Hernández, 2002). 
Dada la heterogeneidad de los participantes y las características temporales y espaciales que definen los entornos virtuales de aprendizaje, la mediación del docente para motivar la participación se hace más necesaria. En este sentido Folgado et al (2020) puntualizan que Los estudiantes en estos entornos requieren mayor motivación y seguimiento de las actividades por parte de los profesores. Enfatizan en la necesidad de que exista un dialogo constructivo entre docentes y alumnos, así como equidad en las oportunidades de participación e interdependencia de los alumnos. Para lograr esto es necesaria una planificación coherente con los diferentes elementos presentes en el proceso educativo, teniendo en cuenta, no solo los objetivos específicos de aprendizaje sino el perfil del grupo. La gran cantidad de herramientas disponibles para generar actividades en línea dan posibilidad al docente de innovar, de potenciar el aprendizaje según los contenidos a impartir y de observar de cerca el comportamiento de cada participante con lo cual puede ofrecerles un trato más efectivo e individualizado.

Con respecto a los contenidos curriculares, Coll, Pozo, Sarabia y Valls (como se citó en Diaz-Barriga y Hernández, 2002), indican que en todos los niveles educativos estos contenidos pueden agruparse en tres áreas: "conocimiento declarativo, procedimental y actitudinal" (p. 52). Partiendo de la definición que Díaz-Barriga y Hernández (2002) hacen de cada uno de estos aprendizajes, se presentan a continuación algunas herramientas que, el docente puede utilizar para propiciar el aprendizaje de estos contenidos en contextos mediados por tecnologías.

Contenidos declarativos (saber qué). Se refiere al aprendizaje de datos, hechos, conceptos y principios. Lo denominan declarativo porque se declara o conforma por medio del lenguaje. Para este tipo de aprendizaje los EVA proveen herramientas de evaluación automática como los cuestionarios, los cuales presentan diversos estilos de preguntas que pueden ser configuradas para que el sistema evalúe las respuestas de forma automática. Así también hay diversos tipos de juegos que se pueden configurar para que automáticamente evalúen la intervención de cada estudiante. De igual manera se pueden planificar actividades de tipo colaborativo como los glosarios y los wikis

Contenidos procedimentales (saber hacer). Son aquellos que se enfocan en la ejecución de procedimientos, técnicas, habilidades, destrezas, etcétera. Es un aprendizaje práctico porque se basa en la realización de varias acciones u operaciones. La evaluación de este tipo de aprendizaje en los EVA se hace por medio del envío del producto finalizado y/o de evidencias de su elaboración (esto puede ser a través de documentos escritos, imágenes o audiovisuales, así como presentaciones en tiempo real). Es importante destacar que, tanto en actividades presenciales como en actividades mediadas por tecnologías de información, es esencial aclarar de forma detallada "la meta a lograr, la secuencia de acciones a realizar y la evolución temporal de las mismas" (Díaz-Barriga y Hernández, 2002, 54)

Contenidos actitudinales (saber ser). Estos contenidos se enfocan en actitudes y valores que generalmente trascienden lo personal y social. Entre las metodologías que intentan promover el aprendizaje en este campo están "la clarificación de valores y juicio crítico, discusión de dilemas y análisis de casos, comprensión y escritura crítica de textos, aprendizaje cooperativo y situado orientado a la comunidad, desarrollo de habilidades sociales afectivas y de autor 
regulación" (Díaz-Barriga y Hernández, 2002, p. 59). En los EVA una herramienta realmente poderosa para promover y evidenciar este tipo de aprendizaje son los foros en sus diferentes formas, ya que en esencia promueven el dialogo y el intercambio de ideas, permitiendo desde su asincronicidad la posibilidad de intervenciones máscompletas ya que el participante tiene tiempo de sustentar y reflexionar cada una de sus intervenciones.

Retroalimentación de las actividades realizadas. Concebida la evaluación como el medio para monitorear el aprendizaje, se hace imprescindible que, como parte de la evaluación, se ponga especial atención en la retroalimentación. La cual es definida por Shute (2008) como aquella información que se comunica al estudiante con la intención de modificar su comportamiento o forma de pensar para mejorar el aprendizaje de un área de contenido o habilidad. Aunque puede haber diferentes tipos de retroalimentación, en general ésta debe estar enfocada en dar apoyo, ser oportuna y específica. Teniendo en cuenta que tanto las características del estudiante como los aspectos específicos de la tarea pueden intervenir en su eficacia.

Por otra parte, Contreras y Zúñiga (2018) la conciben como "un proceso de comunicación dialógica que genera quien enseña a partir de los resultados de procesos de evaluación de sus estudiantes, donde les entrega sugerencias y comentarios con la intención de que reconozcan sus errores y puedan aprender de ellos" (parr.11). Y García-Jiménez (2015) resalta que la información que se devuelve sobre el programa, la institución, la enseñanza o el aprendizaje es lo que da sentido a la evaluación. Señalando también que el grado de concreción, la complejidad y el nivel de elaboración de la retroalimentación va a depender del fin de la evaluación, es decir si es formativa o sumativa.

Por lo antes dicho el docente desde su rol de mediador debe dedicar especial atención a la devolución que da a sus estudiantes a través de la retroalimentación, ya que ésta puede generar resultados positivos o negativos dependiendo de la manera en la que se aborda, en este sentido Contreras y Zúñiga (2018) mencionan varios aspectos relevantes para que la retroalimentación genere resultados positivos, entre los cuales resaltan la importancia de asumirla como parte de los procesos de enseñanza y evaluación. Por esta razón debe considerarse un lapso de tiempo adecuado tanto para entregar como para que el estudiante haga las mejoras en su trabajo. De igual manera la retroalimentación debe estar centrada en el desempeño de la actividad a evaluar y enfocarse en los aspectos prioritarios para el aprendizaje evaluado

En los EVA el proceso de mediación a través de la retroalimentación cobra mayor importancia dadas las características estructurales que determinan la evaluación de los aprendizajes en estos ambientes. Por esta razón las plataformas tecnológicas que gestionan estos entornos tienen bien definidos los espacios para la retroalimentación, poniendo a disposición del docente diferentes formas de proveerla.

En tal sentido, Mogollón (2004) indica que más allá de ser un proceso de evaluación formativa, la retroalimentación viene a ser una comunicación que da a conocer a los estudiantes 
sus avances y logros a lo largo del proceso de un proceso educativo. Así mismo destaca que una retroalimentación de calidad debe:

- Ser directa cuando está dirigida a un participante en particular;

- Expresar detalladamente las características de la conducta a la que hace referencia;

- Ser específica al momento de referir una situación o momento determinado

- Llegar a los estudiantes de forma inmediata si va a referir a un evento próximo

- demostrar la emoción y sentimiento inherentes a la comunicación

De igual manera destaca que la retroalimentación debe ser objetiva, centrándose en la conducta, las observaciones y descripciones de hechos relacionados con el proceso que se está evaluando. Debe estar siempre enfocada en el receptor: en lo que éste puede utilizar, más que en la cantidad de información que se tiene. Así como en lo que se dice en lugar de por qué se dice. Corresponde al docente, como mediador, no solo procurar que se mantenga una retroalimentación efectiva docente-alumno sino también motivar la retroalimentación entre iguales a través del trabajo colaborativo y la coevaluación.

\section{REFLEXIONES FINALES}

Más allá de los avances tecnológicos, la mediación del docente es la que define el éxito o no de los procesos pedagógicos en los diferentes contextos en los que éste se desarrolle. Sobre todo, en los espacios virtuales, esta mediación debe tenerse muy en cuenta como aspecto central del proceso educativo desde el momento de la planificación y diseño de los contenidos a impartir ya que, a pesar de las múltiples opciones que ofrecen las TIC, las posibilidades de improvisación, comunicación gestual y/o grupal aún no son tan efectivas como en la presencialidad.

En los entornos virtuales de aprendizaje la esencia de la mediación surge de los mismo principios filosóficos y teóricos que promueven las actividades colaborativas en el aula, el aprendizaje significativo y la evaluación como parte de los procesos de enseñanza y aprendizaje.

Todos los cambios que las prácticas docentes han experimentado en los últimos meses a raíz de la pandemia, en los que la virtualidad inevitablemente se ha hecho presente, han resaltado la necesidad de mantener una continua formación docente que facilite y potencie las competencias necesarias para ejercer eficientemente la mediación en estos contextos, promoviendo la participación y a su vez garantizando la calidad del proceso educativo.

Tener bien definida la intencionalidad del curso o programa que se está impartiendo, tener presente la realidad de los participantes para seleccionar contenidos y actividades con significado y guiar a los estudiantes para que los conocimientos adquiridos trasciendan más allá del momento y el contexto educativo son factores fundamentales que se logran a través de la mediación y que deben tenerse presente como parte de la dinámica en los entornos virtuales de aprendizaje. Esto se logra manteniendo informado al grupo, fomentando el dialogo y la reflexión, 
motivando la participación y dando una adecuada retroalimentación. Los software o sistemas de gestión de aprendizaje tienen bien definidos los espacios y las herramientas necesarias para desarrollar estas actividades

La mediación del docente en entornos virtuales de aprendizaje es una labor que demanda mucho tiempo y dedicación, que muchas veces traspasa los límites del entorno virtual y del horario de trabajo, siendo este tiempo algunas veces subestimado al momento de hacer el cálculo de horas dedicadas al curso o programa en cuestión. Por lo cual se hace necesario ajustar a la realidad el cálculo del tiempo dedicado a la mediación en estos espacios educativos. 


\section{REFERENCIAS BIBLIOGRÁFICAS}

Contreras, G y Zúñiga, C. (2018). Concepciones sobre Concepciones sobre retroalimentación del aprendizaje: Evidencias desde la Evaluación Docente en Chile. Rev. Actual. Investig. Educ, 18 (3). http://dx.doi.org/10.15517/aie.v18i3.34327

Chiecher, A. y Donolo, D. (2013). De diálogos e intercambios virtuales. La dimensión social y cognitiva de las interacciones entre alumnos. RUSC, 10 (2), 37-53. https://dialnet.unirioja.es/servlet/articulo?codigo $=4627720$

De Armas, N., Poyeaux, A. y Hernández, E. (2017). Concepción Metodológica de Foros en Entornos Virtuales de Aprendizaje. VII Congreso Virtual Latinoamericano de Calidad en Educación Virtual y a Distancia. https://bit.ly/3Atlj1B

Díaz- Barriga, F. y Hernández, G. (2002). Estrategias docentes para un aprendizaje significativo. (2da ed.). Mc Graw Hill/Interamericana Editores, S.A. de C.V.

El Tiempo. (25 de junio 2020). 67\% de los estudiantes en modalidad virtual no terminan sus estudios. Redacción educación. https://bit.ly/37r20ub

Escobar, N. (2011). La Mediación del Aprendizaje en la Escuela. Acción Pedagógica, 20 (1), 59-73. https://dialnet.unirioja.es/servlet/articulo?codigo=6222147

Fernández, J. (17 de octubre 2020). Las oportunidades que la educación en línea brinda hoy a millones de estudiantes en Iberoamérica. El País. https://bit.ly/3s4flIR

Folgado, J., Palos, P. y Aguayo, M. (2020). Motivaciones, formación y planificación del trabajo en equipo para entornos de aprendizaje virtual. Interciencia, 45 (2). https://bit.ly/2VByZK8

Frankola, K. (2001). Why Online Learners Drop Out [Por qué abandonan los estudiantes en línea]. workforce.com. https://www.workforce.com/news/why-online-learners-drop-out

García Aretio, L. (2019). El problema del abandono en estudios a distancia. Respuestas desde el Diálogo Didáctico Mediado. RIED, 22(1), 245-270. DOI:http://dx.doi.org/10.5944/ried.22.1.22433

García-Jiménez, E. (2015). La evaluación del aprendizaje: de la retroalimentación a la regulación. El papel de las tecnologías. RELIEVE, 21 (2). DOI: http://dx.doi.org/10.7203/relieve.21.2.7546

Lobos, C., Figueroa, I. y Soto, J. (2017). Interacciones pedagógicas en docentes categorizados como básicos y competentes en la evaluación docente: una mirada desde la experiencia de aprendizaje mediado (EAM). Foro Educacional, (29), 67-91. http://ediciones.ucsh.cl/ojs/index.php/ForoEducacional/article/view/782 
Ministerio de Ciencia, Innovación y Universidades, (2019). Datos y cifras del sistema universitario español. Publicación 2018-2019. Gobierno de España. https://bit.ly/3fMLU2S

Mogollón, I. (2004). El chat yotros procedimientos de evaluación a distancia aplicables en sistemas mixto. Pixel-Bit, 23, 43-54. https://recyt.fecyt.es/index.php/pixel/article/view/61240/37254

Moore, M. G. (1997). Theory of transactional distance. En Theoretical principles of distance education. Routledge. 22-38. http://www.c3l.uni-oldenburg.de/cde/found/moore93.pdf

Orrú, S. (2003). Reuven Feuerstein y la Teoría de la Modificabilidad Cognitiva Estructural. Revista de Educación, (332), 33-54. https://dialnet.unirioja.es/servlet/articulo?codigo=776714

Ruiz, M., Diestro, A. y García, M. (2016). Participación en foros virtuales en cursos masivos (UNED). REDIE, $\quad 18 \quad$ (3). $\quad$ http://www.scielo.org.mx/scielo.php?pid=S160740412016000300009\&script=sci_arttext

Sánchez, M. (2010). Técnicas docentes y sistemas de evaluación en educación superior. Nerea

Shute, V. (2008). Focus on Formative Feedback. Review of Educational Research, 78(1), 153 -189. doi:10.3102/0034654307313795

Silva, J. (2011). Diseño y moderación de entornos virtuales de aprendizaje (EVA). Editorial UOC. https://bit.ly/37r29hd

Silva, J. (2017). Un modelo pedagógico virtual centrado en las E-actividades. RED, (53). DOI: http://dx.doi.org/10.6018/red/53/10

Soto, A., Briseño, M. y Gómez Zermeño, M. G. (2015). Factores de deserción de cursos virtuales para formación docente en Costa Rica. Revista $Q, 10$ (19). DOI: 10.18566/revistaq.v10n19.a03

Taylor-Guy, P. y Chase, A. (2020). Universities need to train lecturers in online delivery, or they risk students dropping out. The Conversation. https://bit.ly/3ytizSh

Valencia, T., serna, A., Ochoa, S., Caicedo, A., Montes, J. y Chávez, J. (2016). COMPETENCIAS Y ESTÁNDARES TIC desde la dimensión pedagógica: una perspectiva desde los niveles de apropiación de las TIC en la práctica educativa docente. UNESCO. https://bit.ly/2Xed1xr

Veitya, M. (2016). Los foros virtuales, espacios para enriquecer los procesos de argumentación en los estudiantes de posgrado. RICSH, 5 (10). https://www.redalyc.org/pdf/5039/503954317016.pdf 